\title{
Emergence of Quintet Superfluidity in the Chain of Partially Polarized Spin-3/2 Ultracold Atom
}

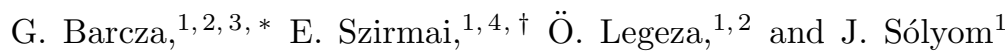 \\ ${ }^{1}$ Wigner Research Centre for Physics, Hungarian Academy of Sciences, H-1525 Budapest, Hungary \\ ${ }^{2}$ Fachbereich Physik, Philipps Universität, G-35032 Marburg, Germany \\ ${ }^{3}$ Department of Physics, Loránd Eötvös University, H-1518 Budapest, Hungary \\ ${ }^{4}$ BME-MTA Exotic Quantum Phases "Lendület" Research Group, \\ Budapest Univ. of Technology and Economics, Institute of Physics, H-1111 Budapest, Hungary
}

(Dated: January 19, 2012)

\begin{abstract}
The system of ultracold atoms with hyperfine spin $F=3 / 2$ might be unstable against the formation of quintet pairs if the interaction is attractive in the quintet channel. We have investigated the behavior of correlation functions in a model including only $s$-wave interactions at quarter filling by large-scale density matrix renormalization group simulations. The correlations of quintet pairs remain short-ranged even for strong attractive interaction in the paramagnetic ground state. They become, however, quasi-long-ranged when the system is partially polarized, in a broad range around two thirds of saturation. We also discuss possible experimental realizations and detection of quintet pairing in the system of ultracold atoms.
\end{abstract}

PACS numbers: $71.30 .+\mathrm{h}, 71.10 . \mathrm{Fd}$

Recently ultracold atomic and molecular systems have been in the focus of theoretical and experimental studies not only in atomic and molecular physics but also in condensed matter physics [1]. This is due to the fact that these systems provide controllable simulators of complicated condensed matter systems. It is expected that they will provide answers for a series of long-standing questions related to topics like Mott transition, BEC to BCS crossover, non-Fermi liquid behavior of high- $T_{c}$ superconductors, or anomalous quantum Hall effect. Moreover, atomic systems with hyperfine spin degrees of freedom higher than $1 / 2$ can show completely new behavior for both bosonic and fermionic systems. For repulsive interaction various Mott insulating phases, (chiral) spin liquid states, resonating plaquette order, spin-quadrupole and even higher multipole order [2] can occur in two- or threedimensional systems, while in one dimension a generalized Peierls-like distortion can characterize the ground state with oscillating bond-order parameter [3]. For attractive interactions bound trionic and quartet states have been predicted for one- and three-dimensional systems as well [4].

In addition to the usual singlet BCS pairs, nonsinglet pairs can also occur in principle if the appropriate component of the interaction is attractive. Due to symmetry reasons, spin-triplet pairs of spin- $1 / 2$ electrons can exist only in $p$-wave - or more generally in odd- $l$-wave - superconductors, while $s$-wave pairs of higher spin fermions can also be in a nonsinglet spin state, e.g., $F=3 / 2$ fermions may form spin-2 (quintet) pairs. These states are of particular interest for their exotic properties especially in magnetic field [5].

For a long time magnetic ordering and superconductivity were thought to be incompatible. In fact homogeneous ferromagnetic order does not allow a homogeneous superconducting order of singlet Cooper pairs. Coexistence is possible for $p$-wave triplet pairs [6] or in inhomogeneous singlet superconductivity with finite momentum of the pairs, i.e., in the Fulde-Ferrell-Larkin-Ovchinnikov (FFLO) phase [7].

In this Letter, we study the possible formation of local quintet pairs and their stability in a one-dimensional chain of fermionic atoms with hyperfine spin $F=3 / 2$, when the interaction is attractive in the quintet channel. We consider a quarter-filled system, namely, the number of particles is equal to the number of sites. We show that although correlation functions of quintet pairs decay exponentially in the paramagnetic ground state, the quintet pairs can be stabilized by a strong enough external magnetic field due to population imbalance of the particles with different spin components. The correlation of quintet pairs shows algebraic decay and quintet pairing becomes the dominant instability in the system. This can occur in a wide range of the magnetic field. The main advantage of the presented model is that it contains only $s$-wave interaction and the emergence of quintet pairing does not require $p$-wave Feschbach resonance, thus experimental realization of non-singlet pairs becomes much easier.

The scattering processes between the scattering particles with hyperfine spin $F$ can be classified into independent spin channels characterized by the total spin $(S)$ of the two scattered atoms: $V=\sum_{S=0}^{2 F} g_{S} P_{S}$, where $P_{S}$ projects onto the total spin $S$ subspace and $g_{S}$ is the coupling constant in the corresponding channel. The $g_{S}$ is related to the $a_{S} s$-wave scattering length in the total spin $S$ scattering channel as $g_{S}=\frac{4 \pi \hbar^{2} a_{S}}{\nu}$ with $\nu$ atomic mass. The projectors are expressed via the pairing operators as $P_{S}=\sum_{m, i} P_{S m, i}^{\dagger} P_{S m, i}$ which are defined through the Clebsch-Gordan coefficients [9] and 
the $c_{\alpha, i}^{\dagger}$ spin- $\alpha$ fermion creation operator as $P_{S m, i}^{\dagger}=$ $\sum_{\alpha, \beta}\left\langle\frac{3}{2}, \frac{3}{2} ; \alpha, \beta \mid S, m\right\rangle c_{\alpha, i}^{\dagger} c_{\beta, i}^{\dagger}$, where $m$ is the $z$ component of the total spin of the two scattering particles. Starting from a fermionic spin-3/2 Hubbard-like model with on-site interaction, the only contributing terms are antisymmetric under the exchange of the spin of the two colliding atoms, therefore, only the $S=0$ and $S=2$ terms may appear. Thus the Hamiltonian of the system reads as

$$
\mathcal{H}=-t \sum_{i, \alpha}\left(c_{\alpha, i}^{\dagger} c_{\alpha, i+1}+H . c .\right)+g_{0} P_{0}+g_{2} P_{2},
$$

where $t$ measures the overlap between neighboring sites. For attractive couplings $\left(g_{0}<0, g_{2}<0\right)$ the above Hamiltonian suggests that singlet and quintet pairs are competing. The region with repulsive interaction in the singlet channel $\left(g_{0}>0\right)$ turned out to be more relevant for quintet pairing. We will therefore consider the case $g_{0}>0, g_{2}<0$, where quintet pairing competes with density waves, as seen if the Hamiltonian (11) is rewritten in terms of the density, $n_{i}=\sum_{\alpha} n_{\alpha, i}=\sum_{\alpha} c_{\alpha, i}^{\dagger} c_{\alpha, i}$, and the $P_{2}$ quintet projector as

$$
\mathcal{H}=-t \sum_{i, \alpha}\left(c_{\alpha, i}^{\dagger} c_{\alpha, i+1}+H . c .\right)+\frac{U}{2} \sum_{i} n_{i}^{2}+V P_{2},
$$

with couplings $U=2 g_{0}, V=g_{2}-g_{0}$.

First we have studied the stability of quintet pairs in the simplest way, i.e., within a self-consistent mean-field approximation. Due to the competing terms in Hamiltonian (2) we supposed site-centered quintet pairs and atomic density wave characterized by the pairing order parameters $\left\langle P_{2 m, i}^{\dagger}\right\rangle(m=-2, \ldots, 2)$ and $\left\langle n_{i}\right\rangle$, respectively. Since the atoms have four different internal states in spin- $3 / 2$ systems, it is natural to assume a maximum four-fold periodicity at quarter filling. Accordingly we considered a 4-sublattice ansatz to solve the self-consistent mean-field equations and to determine the mean-field phase diagram. Two different stable phases are found in the quadrant $g_{0}>0, g_{2}<0$ separated by a line lying around $g_{2}=-1$ (in units of the hopping parameter $t$ ). For weak attractive quintet couplings $\left(-1<g_{2}<0\right)$ the system is in the Mott phase: $\left\langle n_{i}\right\rangle=2$ on odd sites and $\left\langle n_{i}\right\rangle=0$ on even sites, or vice versa. For stronger attractive quintet couplings the atomic density oscillates in the same way as in the other phase, however, in addition to that the quintet pairing order parameter $\left\langle P_{20, i}^{\dagger}\right\rangle$ becomes finite, i.e., local quintet pairs with $m=0$ are formed on every other site.

This mean-field phase diagram turns out to be incorrect for one-dimensional systems. Analytic calculation in the weak-coupling limit [10] shows that the leading instability for $g_{0}>0, g_{2}<0$ is the formation of site- or bond-centered quartets. In order to search for possible conditions that might stabilize the quintet Cooper-pairs in one dimension we have studied numerically the phase diagram of model (11) for $g_{2} \leq 0$ at quarter filling using the density matrix renormalization group (DMRG) method [8]. The simulations have been performed with open boundary condition up to $L=64$ sites, keeping $500-1000$ block states and using up to 8 sweeps. Properties of the various phases have been determined by analyzing the decay of several correlation functions, like different pairings, quarteting, density and spin correlation functions, respectively: $\chi_{S m}(i)=\left\langle P_{S m, 1}^{\dagger} P_{S m, 1+i}\right\rangle$, with $m=0, \pm 1, \pm 2, \chi_{Q}(i)=\left\langle Q_{1}^{\dagger} Q_{1+i}\right\rangle, \chi_{n}(i)=$ $\left\langle n_{1} n_{1+i}\right\rangle-\left\langle n_{1}\right\rangle\left\langle n_{1+i}\right\rangle, \chi_{\tilde{m}}(i)=\left\langle\tilde{m}_{1} \tilde{m}_{1+i}\right\rangle-\left\langle\tilde{m}_{1}\right\rangle\left\langle\tilde{m}_{1+i}\right\rangle$, where $\tilde{m}_{i}=\sum_{\alpha} \alpha n_{\alpha, i}$ and the operator to study quarteting is $Q_{i}^{\dagger}=c_{3 / 2, i}^{\dagger} c_{1 / 2, i}^{\dagger} c_{-1 / 2, i}^{\dagger} c_{-3 / 2, i}^{\dagger}$. The analysis of the above defined correlation functions confirmed the absence of quintet Cooper pairs in 1D system in the absence of magnetic field, since the quintet pairing correlations $\left(\chi_{2 m}\right)$ decay exponentially for all $m$ in the whole quadrant $g_{0}>0, g_{2}<0$. We have found, in agreement with Ref. [10], that $\chi_{Q}$ and $\chi_{n}$ show algebraic decay in the regime where site-centered quartet phase was predicted by weak coupling analysis.

The spin singlet quartets which are formed from an equal number of atoms with spin components: $\alpha=$ $3 / 2,1 / 2,-1 / 2$ and $-3 / 2$ could be broken and quintet pairs could be stabilized, if a population imbalance occurs in the number of fermions with different spin components. This can be achieved, for example, by switching on a magnetic field $(B)$ which couples linearly to the magnetization $\tilde{m}=\frac{1}{L} \sum_{i}\left\langle\tilde{m}_{i}\right\rangle$ measured in units of Bohr magneton. Therefore, we have calculated the correlation functions for increasing magnetization from $\tilde{m}=0$ up to the maximum value $\tilde{m}=3 / 2$.

Before presenting the results, it is interesting to see how the number of atoms with spin components $\alpha=$ $-3 / 2,-1 / 2$, and $1 / 2$ decreases in increasing field while more and more atoms have $\alpha=3 / 2$. This depends on the interaction between the particles. Performing calculations at several points in the $\left(g_{0}>0, g_{2}<0\right)$ quadrant we have found two types of dependence of $\left\langle n_{\alpha, i}\right\rangle$ on $\tilde{m}$ as displayed in Fig. 1. The regions, where one or the other behavior is realized, correspond roughly to the regions, where the site-centered or the bond-centered phase is the $\tilde{m}=0$ ground state [10]. The pictograms at $\tilde{m}=0$ in Fig. 1 indicate these states. The transition between the two types of behaviors occurs roughly around $g_{0}=-3 g_{2}$.

For $\tilde{m} \geq 1$ the model becomes independent of $g_{0}$ due to the absence of fermions with $\alpha=-3 / 2$ and $-1 / 2$ in both regions. The quintet correlation function $\chi_{22}$ shows an algebraic decay as shown in Fig. 2, while all other $\chi_{2 m}$ are zero. The Fourier spectrum of $\chi_{22}$ has a peak at $\pi$, which indicates two-site periodicity of the quintet correlations, although, the particle density remains homogeneous in agreement with the expectations in one dimension. This spatial correlation is illustrated by the pictograms in Fig. 1 local quintet pairs of atoms with $\alpha=3 / 2$ and $1 / 2$ 


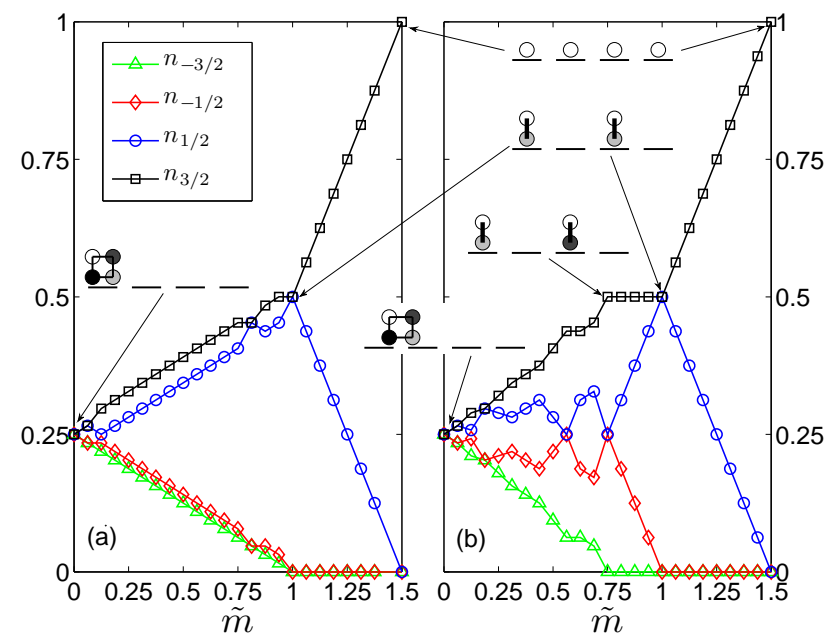

FIG. 1. (Color online) Density of the fermions with different spin component vs. magnetization $\tilde{m}$ for a chain with $L=64$ sites (a) at $g_{0}=2, g_{2}=-4$, and (b) at $g_{0}=20, g_{2}=$ -4 . The pictograms illustrate the spatial structure of the quarteting, pairing quintet and ferromagnetic phases (see the text for the details). Shading of the circles corresponds to the different $\alpha$ hyperfine spin components of the atoms, and the lines connecting the atoms indicate that the atoms form pairs and quartets with finite anomalous expectation values: $\left\langle c_{\alpha}^{\dagger} c_{\beta}^{\dagger}\right\rangle$ and $\left\langle c_{\alpha}^{\dagger} c_{\beta}^{\dagger} c_{\gamma}^{\dagger} c_{\delta}^{\dagger}\right\rangle$, respectively.

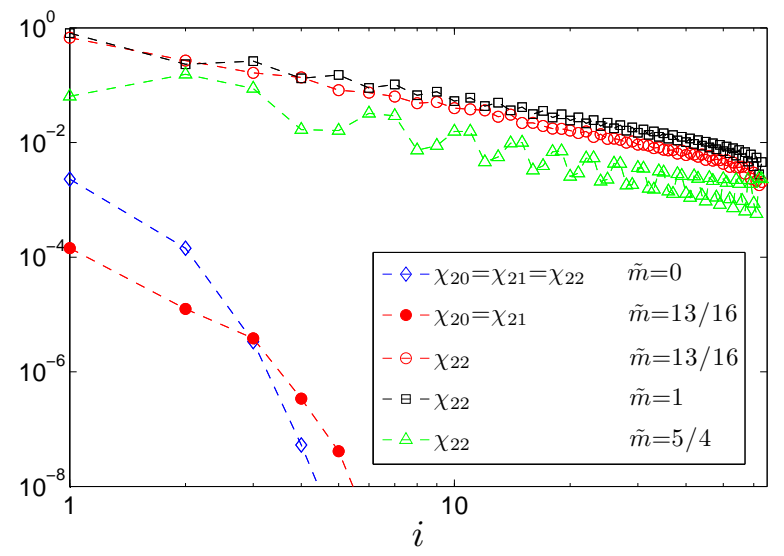

FIG. 2. (Color online) Decay of the quintet correlation functions $\chi_{2 m}$ for $\tilde{m}=0, \tilde{m}=13 / 16, \tilde{m}=1$ and $\tilde{m}=5 / 4$, calculated for $g_{0}=2, g_{2}=-4$. For the length of the chain $L=64$.

can be formed at quarter filling only on every other site. In addition, $\chi_{n}$ and $\chi_{\tilde{m}}$ decay algebraically with the same periodicity as $\chi_{22}$, but $\chi_{n}(i)=\chi_{\tilde{m}}(i)=(-1)^{i} \chi_{22}(i)$. As the imbalance of the two remaining spin components is increased, and the weight of the $\alpha=1 / 2$ spin component decreases, the $m=2$ quintet pairs start to dissolve and a ferromagnetic background appears. Nevertheless, the quintet pairing $\left(\chi_{22}\right)$ is the most slowly decaying correlation function for a broad region of $\tilde{m}$ (see Fig 2), although its periodicity becomes incommensurate with the lattice, and the leading Fourier component of $\chi_{22}$ shifts from $\pi$ towards 0 . The system becomes ferromagnetically ordered at $\tilde{m}=3 / 2$, and the Fourier spectrum has a single peak at $q=0$. This behavior is in complete agreement with the results of Batrouni et al. [11], since our model can be mapped exactly to their model when $\tilde{m} \geq 1$. This FFLO phase has also been studied in two component, spin polarized systems both theoretically [12] and experimentally [13].

In contrast to this, the population imbalance of fermions with different spin components shows markedly different character in the two regions for $\tilde{m}<1$. For $g_{0}<-3 g_{2}$ (Fig. 11) all spin components start to gain finite weight for $\tilde{m}<1$. As a consequence, the density of the spin quintet pairs starts to decrease, but $\chi_{22}$ remains the slowest decaying correlation function in the system at least when $\tilde{m}>3 / 4$. Although, it is difficult to distinguish between an exponential or algebraic decay of the correlation functions below $\tilde{m} \approx 3 / 4$, the quintet pairing is still the dominant instability even slightly below $\tilde{m}=3 / 4$. Due to the emergence of the paramagnetically favoured spin-singlet quartets, the leading Fourier component of $\chi_{22}$ shifts towards $\pi / 2$. Even though all spin components have finite weight, the other correlation functions $\chi_{2 m}$ with $m=0, \pm 1$, and -2 decays exponentially (Fig. 2) for $\tilde{m}<1$.

A different behavior is found for $g_{0}>-3 g_{2}$ (Fig. 1b). As the polarization decreases from $\tilde{m}=1$ to $3 / 4$, the density of atoms with $-3 / 2$ spin component remains zero, half of the atoms have $\alpha=3 / 2$, while the density of atoms with $\alpha= \pm 1 / 2$ varies linearly with $\tilde{m}$ becoming equal at $\tilde{m}=3 / 4$. In the whole range $\chi_{2,-2}$ and $\chi_{2,-1}$ are equal to zero due to the missing $\alpha=-3 / 2$ spin component and $\chi_{20}$ decays exponentially. On the other hand, the slowest decaying correlation functions, $\chi_{21}$ and $\chi_{22}$ show algebraic decay with identical exponent as shown in Fig. 3. As $\left\langle n_{-1 / 2}\right\rangle$ is increasing, the density of the $m=1$ pairs also increases and the number of the $m=2$ pairs decreases. The amplitude of $\chi_{21}$ increases accordingly and the leading Fourier-component of the $\chi_{22}$ and $\chi_{21}$ correlation functions shifts from $\pi$ toward $\pi / 2$. This spatial correlation can be illustrated as if quintet pairs of $\alpha=3 / 2$ and $\alpha=1 / 2$ atoms were alternating with quintet pairs of $\alpha=3 / 2$ and $\alpha=-1 / 2$ atoms as shown in Fig. 10. Therefore, the one-component quintet pairing found for $\tilde{m} \geq 1$ is replaced by a two-component quintet pairing for $\tilde{m}<1$. We have found that the $m=1$ and $m=2$ quintet pairing remain the dominant instability also for $\tilde{m}<3 / 4$ at least for strong enough imbalance. For smaller values of $\tilde{m}$ all $\chi_{2 m}$ decay exponentially (Fig. 2) due to the emergence of the paramagnetically favoured spin-singlet quartets and the leading Fourier component of $\chi_{2 m}$ remains at $\pi / 2$.

Spin-3/2 fermionic condensation could be realized experimentally not only in $3 \mathrm{D}$ but in $1 \mathrm{D}$, too, using ${ }^{132} \mathrm{Cs}$, ${ }^{9} \mathrm{Be},{ }^{135} \mathrm{Ba},{ }^{137} \mathrm{Ba}[1]$. Since according to the results 


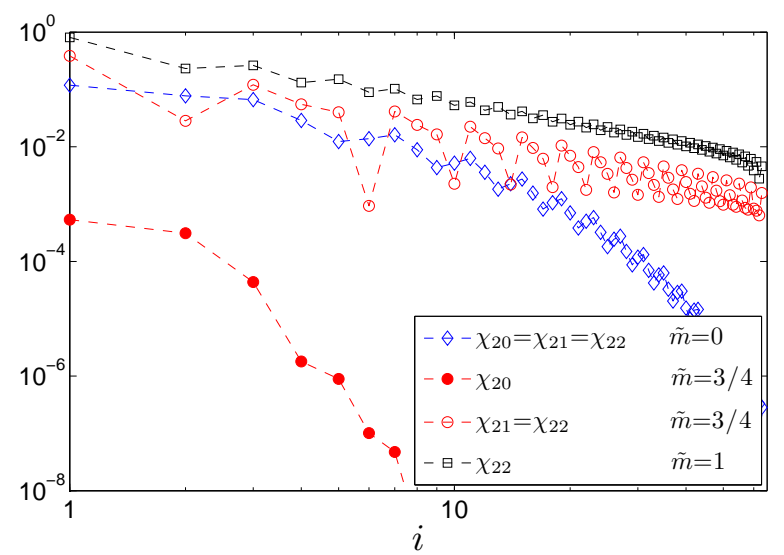

FIG. 3. (Color online) Decay of the quintet correlation function $\chi_{2 m}$ for $\tilde{m}=0,3 / 4$ and 1 calculated at $g_{0}=20, g_{2}=-4$.

presented above quintet pairs exists in a wide range of magnetization for attractive quintet interaction, the experimental realization of the quintet superfluid does not require fine tuning in the $\left(g_{0}, g_{2}, \tilde{m}\right)$ parameter space. In addition, the presented quintet pairing phases can be stabilized via $s$-wave Feschbach resonance avoiding the difficulties with inelastic loss of $p$-wave scatterings. To detect the $m=1$ and $m=2$ quintet pairs, it is crucial to probe the pairing and to measure the underlying magnetic order. There are several possibilities to probe these many-body correlation effects, for instance, pairing can be probed by time-of-flight absorption image [15] or the pair gap can be studied by radio-frequency spectroscopy [16] or momentum-resolved Bragg-spectroscopy [17. In addition, magnetic excitations can also be observed by quantum-noise spectroscopy [18]. Elastic Bragg-spectroscopy [19] can also be applied to detect the variation of the periodicity of the quintet pairs.

In this work, we have investigated the competition between density wave and pair formation in the system of $F=3 / 2$ cold atoms in one-dimensional optical traps at quarter filling. The behavior of various correlation functions has been studied via large-scale, high precision DMRG simulations. We have found that external magnetic field can stabilize different quintet pair states when the spin-population imbalance of fermions breaks up the singlet quartets. While we have found an FFLO-like state of $m=2$ quintet pairs for large population imbalance, two-component superfluidity with both $m=2$ and $m=1$ pairs characterizes the system for intermediate values of the magnetization. Since our model contains only $s$-wave interaction, this result opens a new possibility for the experimental realization and detection of nonsinglet pairing with ultracold atomic systems. We also suggest that in higher dimensional systems, where a mean-field solution might be more reliable, and quintet pairs may exist even in the paramagnetic case, external field can further stabilize them.
This research was supported in part by the Hungarian Research Fund (OTKA) under Grant No. K68340 and K73455. Ö. L. acknowledges support from the Alexander von Humboldt foundation. The authors acknowledge computational support from the Philipps Universität, Marburg and the Dynaflex Ltd.

* electronic address: barcza.gergely@wigner.mta.hu

$\dagger$ electronic address: eszirmai@gmail.com

[1] M. Lewenstein, et al., Adv. Phys. 56, 243 (2007).

[2] K. Harada, N. Kawashima, and M. Troyer, Phys. Rev. Lett. 90, 117203 (2003); C. Honerkamp and W. Hofstetter, Phys. Rev. Lett. 92, 170403 (2004); M. A. Cazalilla, A. F. Ho and M. Ueda, New J. Phys, 11103033 (2009); M. Hermele, V. Gurarie, and A. M. Rey, Phys. Rev.Lett. 103, 135301 (2009); A. V. Gorshkov et al., Nature Physics 6, 289 (2010); E. Szirmai, M. Lewenstein, EPL 93, 66005 (2011); H. H. Tu, G. M. Zhang, and L. Yu, Phys. Rev. B 74, 174404 (2006).

[3] K. Buchta, Ö. Legeza, E. Szirmai, and J. Sólyom Phys. Rev. B 75, 155108 (2007); E. Szirmai, Ö. Legeza, and J. Sólyom, Phys. Rev. B 77, 045106 (2008); S. R. Manmana, et al, Phys. Rev. A 84, 043601 (2011).

[4] Á. Rapp, et al, Phys. Rev. Lett. 98, 160405 (2007); X. W. Guan, M. T. Batchelor, C. Lee, and J. Y. Lee, EPL 86, 50003 (2009).

[5] C. Wu, Int. J. Mod. Phys. B 24, 311 (2010).

[6] S. S. Saxena et al., Nature 406, 587 (2000); C. Pfleiderer et al., Nature 412, 58 (2001).

[7] P. Fulde and R. A. Ferrell, Phys. Rev. 135, A550 (1964); A. I. Larkin and Yu. N. Ovchinnikov, Sov. Phys. JETP 20, 762 (1965).

[8] S. R. White, Phys. Rev. Lett. 69, 2863 (1992); Phys. Rev. B 48, 10345 (1993); U. Schollwöck Rev. Mod. Phys. 77, 259 (2005).

[9] The explicit form of the pairing operators is

$$
\begin{gathered}
P_{00, i}^{\dagger}=c_{3 / 2, i}^{\dagger} c_{-3 / 2, i}^{\dagger}-c_{1 / 2, i}^{\dagger} c_{-1 / 2, i}^{\dagger} \\
P_{20, i}^{\dagger}=c_{3 / 2, i}^{\dagger} c_{-3 / 2, i}^{\dagger}+c_{1 / 2, i}^{\dagger} c_{-1 / 2, i}^{\dagger} \\
P_{2, \pm 1, i}^{\dagger}= \pm \sqrt{2} c_{ \pm 3 / 2, i}^{\dagger} c_{\mp 1 / 2, i}^{\dagger} P_{2, \pm 2, i}^{\dagger}= \pm \sqrt{2} c_{ \pm 3 / 2, i}^{\dagger} c_{ \pm 1 / 2, i}^{\dagger}
\end{gathered}
$$

[10] C. Wu, Phys. Rev. Lett. 95, 266404 (2005); S. Capponi, et al., Phys. Rev. B 75, 100503(R) (2007); G. Roux, S. Capponi, P. Lecheminant, P. Azaria, Eur. Phys. J. 68, 293 (2009).

[11] G. G. Batrouni, M. H. Huntley, V. G. Rousseau, and R. T. Scalettar, Phys. Rev. Lett. 100, 116405 (2008).

[12] K. Yang, Phys. Rev. B 63, 140511(R) (2001); G. Orso, Phys. Rev. Lett. 98, 070402 (2007); M. Rizzi et al., Phys. Rev. B 77, 245105 (2008); A. Lüscher, R. M. Noack, and A. M. Läuchli, Phys. Rev. A 78, 013637 (2008).

[13] T. Mizushima, K. Machida, and M. Ichioka, Phys. Rev. Lett. 94, 060404 (2005); Y. Liao, et al, Nature 467, 567 (2010).

[14] C. Ospelkaus, et al., Phys. Rev. Lett. 97, 120402 (2006), T. Stöferle, H. Moritz, K. Günter, M. Köhl, and T. Esslinger, Phys. Rev. Lett. 96, 030401 (2006).

[15] W. Ketterle and M. W. Zwierlein, Proceedings of the International School of Physics "Enrico Fermi" IOS Press, 2007. 
[16] C. A. Regal, C. Ticknor, J. L. Bohn, and D. S. Jin, Nature 424, 47 (2003); C. Chin, et al., Science 305, 1128 (2004).

[17] P. T. Ernst, et al., Nature Physics 6, 56 (2010).

[18] G. M. Bruun, B. M. Andersen, E. Demler, and A. S. Sorensen, Phys. Rev. Lett. 102, 030401 (2009); K. Eck- ert, et al., Nature Physics 4, 50 (2008); E. Altman, E. Demler, and M. D. Lukin, Phys. Rev. A 70, 013603 (2004).

[19] D. van Ooesten et al., Phys. Rev. A 71, 021601 (2005). 